\title{
Making Visual Maps Accessible to the Blind
}

\author{
Maria Claudia Buzzi, Marina Buzzi, Barbara Leporini* and Loredana Martusciello
}

\author{
CNR-IIT, via Moruzzi 1, 56124 Pisa, Italy \\ ${ }^{*}$ CNR-ISTI, via Moruzzi 1, 56124 Pisa, Italy \\ \{Claudia.Buzzi, Marina.Buzzi, Loredana.Martusciello\}@iit.cnr.it, \\ Barbara.Leporini@isti.cnr.it
}

\begin{abstract}
Visual maps deliver content in a simple and effective way. They can be useful for various purposes in areas such as street guidance, surrounding information, and education. However, sightless persons are unable to explore visual maps and risk being cut off from several multimedia Web applications. In this paper, starting from accessibility issues of map-based applications, we discuss possible interaction modalities and devices to use for truly achieving usage perspectives desired by blind people. New ways to interact with a mobile device (such as gestures or touch commands) and possible solutions for making a map truly effective are investigated. In order to better explain the issues and needs of blind users accessing visual maps, an example of an interaction is presented.
\end{abstract}

Keywords: Accessibility, blind, visual maps, mouse, touch screen

\section{Introduction}

Today's Web is increasingly rich in multimedia and dynamic content. Anybody with a computer or a smart phone can produce content, access social networks or explore dynamic content. Visual maps are especially useful for traveling and locating places rapidly and effectively. Google Maps (http://maps.google.com/) is one of the most popular tools showing street maps, satellite and 3D images. It is often linked by organizations (hotels, museums, conference sites, shops, public offices, service providers, etc.) to offer their customers or visitors simple-to-use directions with the "Get directions" function for tracing routes (by car, bus, walking, or bike) between one's starting point and one or more destinations. Likewise, several online services such as booking websites (e.g., hotel reservations) use visual maps to locate the place or to explore its surroundings. Exploring maps could also be useful for blind children, as a tool for effectively studying geography as well as geometry.

Unfortunately, these visual-based contents do not fully benefit a blind person interacting with the computer via screen reader, voice synthesizer and keyboard, since the graphic part of the information is lost.

Map exploration has three main application fields: 1) for orientation and movement, 2) for exploration and localization of points of interest, and 3) for educational purposes. A previous study showed that tactile sense in a blind child 
develops links between neurons in the brain area that in a sighted person is dedicated to sight. This suggests that the function of touch may replace (in the sense of being equivalent, performing the same function) the perception acquired by experiencing sight [1].

The problem of delivering an equivalent description for any non-text content is one of the main guidelines of the WCAG 2.0 [2]. Satisfying this requirement ensures the same content or function will be delivered to individuals with reduced perception. The real issue is how to enable visually-impaired users to obtain the same information provided by a visual map. Indeed, it is important not only to provide alternative descriptions to the graphical elements, but also to find a solution aimed at putting the user in the same conditions as a sighted person. However, achieving this equivalence is a big challenge and new approaches are needed to enhance interaction and reduce the integration gap between normally- and differently-abled persons. Visual maps offer various opportunities. All modalities must be exploited to provide the same information and functionalities to visually-impaired users, and make maps accessible to and truly usable by all.

In this paper we discuss issues related to potential applications based on accessible maps as well as possible techniques and devices for achieving our goal. A common case is how to access maps via screen reader, or through assistive technologies on a mobile device. Particular consideration will be given to possible new ways to interact with a mobile device, such as gestures or touch commands. The discussion in this paper could be a valuable analysis for identifying user requirements for visual map accessibility. In addition, possible solutions for making a map really effective will be suggested. In order to better explain the issues and needs of blind users accessing visual maps, an example of interaction will be presented. Touch screens, advanced mobile devices or well-structured online maps can greatly increase opportunities for people with impaired vision.

\section{Related Works}

Most maps today have legal or technical restrictions on their use, preventing people from using them in creative, productive, or unexpected ways. The OpenStreetMap project was created to fulfill this requirement: it provides free geographic data such as street maps to anyone who wants them [3]. Related to this project, blind.accessiblemaps.org is trying to develop an alternative system that provides geographic data, also keeping in mind accessibility for blind persons. The project, still in a beta version, proposes the use of special tags of interest on visual maps, for visually impaired users (i.e.: "Traffic Signals with sound for walk").

Images are used everywhere for many purposes such as logos and branding, signage, adverts, photos and illustrations. Some images are designed to be purely aesthetically pleasing, many are designed to convey information of some type, but all are potentially interesting to individuals with sight loss. For these reasons it is important to make graphic information accessible for all. There are a few ways to make this graphical information accessible, and the most appropriate depend on the requirements of the individual or user group concerned. Image descriptions are intended to replace a given image by conveying the core message in descriptive text 
or audio. Large print images are intended to be used by sighted or partially sighted people, and can be as simple as very clear versions of the print original. Tactile graphics are images which are specially created to be touched rather than looked at. There are a number of ways to produce tactile graphics. However, converting a visual graphic to an appropriate tactile graphic is not simply a matter of taking a visual image and making some kind of "tactile photocopy". The tactile sense is considerably less sensitive than the visual sense, and touch works in a more serial manner than vision. Therefore the visual graphic needs to be re-designed to make sense in a tactile form for blind and partially sighted readers.

In the museum environment, tactile exploration of reproductions of famous paintings in three dimensions (bas reliefs made of special material) might be an alternative technique for conveying visual information to visually impaired people (e.g. http://www.cavazza.it/museoanteros/). This is an interesting approach but it is costly and difficult to scale. New solutions that are cheaper and easy to implement are still needed. A recent approach by Miao et al. uses tactile paper prototyping to bridge the visual and haptic modality while ensuring multimodality when a screen reader user accesses graphical user interfaces [4].

Tactile maps can support blind people's mobility but to enjoy full independence they need to generate tactile maps by themselves, to easily check their walking route anytime as needed. [5]. To improve the accessibility of geographic data, Zeng et al. developed an audio-haptic map browser to access GIS data through a large-scale Braille display. The haptic map system supports interactive multimodal interaction by combining audio and haptic feedback. Furthermore, gesture recognition of fingers establishes a natural interaction for navigation of tactile maps [6].

In 1985, in a short unpublished paper, Loomis [7] had proposed the idea of developing a system of digital maps as part of a navigation system for the visually impaired, with audio information indicating the user's position, possible interesting places and the best route to follow to reach them. Today many interests focus on multimodal devices with audio, visual and tactile output. In the context of haptic devices, the HyperBraille project (http://www.hyperbraille.de/) has been founded by the German Federal Ministry of Economics and Technology, with the aim of developing a touch-sensitive tablet display for blind and partially sighted users. Spindler et al. [8] proposed re-designing existing non-visual approaches and the development of appropriate haptic interaction techniques for tactile widgets. Specifically, they describe a complex user interaction with tactile widgets and how to adapt a screen explorer developed on the HyperBraille project to requirements of third party applications (such as plug-ins).

In the framework of the EU project HaptiMap, Magnusson et al. [9] carried out a study on how to make geospatial information more usable and accessible by the use of a haptic display in combination with audio feedback, suggesting a roadmap for dealing with these challenges (http://www.haptimap.org/). The HaptiMap project is aimed at making maps and location-based services more accessible by using several senses like touch, hearing and vision, with the goal of increasing the number of persons who are able to use mainstream map services. Within the same project, McGookin et al. [10] presented the initial design and development of Virtual Navigator, a 3D virtual haptic (used as a virtual white cane) and audio training 
simulator for route navigation, designed to improve the support provided by mobility trainers to blind and visually impaired cane users in the U.K.

Habel et al. [11] proposed a verbally assisting virtual-environment tactile map (VAVETaM) that utilizes a haptic force-feedback device. The generation of verbal instructions and descriptions are extracted from the user's tactile explorations of the virtual-environment tactile map. The goal of the VAVETaM project is to generate verbal assistance for virtual-environment tactile map exploration, both to communicate labeling information like street and building names and to assist the user's exploration.

To explore digital maps and display them in tactile way, Schmitz et al. [12] developed a system that uses a Braille display or a text-to-speech engine, and a standard rumble gamepad, used to take requests from the user and to give him tactile output. Sánchez [13] presented a set of applications studied for PocketPC devices to assist the navigation of blind users in a real environment; he developed the applications for four different contexts (neighborhood, bus transportation, Metro network and indoor environments such as schools), carrying out a usability evaluation for each of them.

Addressing blind or severely visually impaired travelers' needs, the Tactile Maps Automated Production (TMAP) project [14] combined existing tools such as the World Wide Web, geographic information systems, Braille embossers and touch tablet technology.

Marsto et al. [15] manipulated values of attributes in digital map files produced by TMAP (in the Scalable Vector Graphics - SVG - format) to have visual maps that can present these data in a format usable by people with varying degrees of visual impairment. Kulyukin et al. [16] presented an algorithm for placing street names on street maps produced by TMAP software; the algorithm takes as input the user preferences configured on a XML file and generates a SVG file.

Wagner [17] investigated the use of audio-tactile maps in an educational context with visually impaired users, and proposed the speech- and audio-enhanced tactile map as a possible solution. Wang et al. [18] proposed a solution to automatically convert visual digital maps into interactive tactile-audio maps. The result is a tactile map and an SVG file containing text and graphical information used with a touchpad.

Moreover, gesture interfaces were also investigated in association with handheld devices that offer touch screens. Brock et al. [19] investigated the use of multi-touch displays for multimodal interactive maps to use gestural interaction; they proposed a solution to improve accessibility of geographical information for blind users, with multimodal interactive maps obtained by placing a tactile map on a touch device. Paladugu et al. [20] proposed and evaluated a set of patterns with different shapes and textures, to use for adaptive map rendering via a legend; they suggested putting a start information button on the top right corner of the map to help blind users to find the route start/end points by touch.

Making geo-referenced information accessible using multimodal interfaces (e.g. audio and haptic), the project Atlas.txt [21] investigated how geo-referenced information (often conveyed via shaded thematic choropleth maps) can be communicated to blind users; the project developed a prototype data-to-text Natural Language Generation (NLG) system that produces textual summaries of UK 2001 Census data. 
Concerning accessibility of touchscreens, McGookin et al. [22] carried out a study that illustrates accessibility problems on touchscreen use for visually impaired users, comparing two different methods for confronting this problem: to overlay a raised paper control panel that incorporated tactile buttons on the screen and to use a touchscreen gesture-based player. Some guidelines are provided to design more accessible interfaces for future touchscreens.

Crossan et al. [23] proposed a non-visual browsing mechanism where users can run their fingers over a touchscreen to feel a vibrotactile texture depending on the functionality of that area of the touchscreen, with the use of a vibration motor on the back of the mobile device. Lastly, not strictly addressed to visually impaired people, McGookin et al. [24] describe two interaction techniques to display off-screen points of interest in maps shown on mobile visual displays [25].

\section{Discussion}

As mentioned in the Introduction, maps can be used for various purposes, such as finding a target or exploring the surroundings, or for educational aims, such as understanding shapes and distances. Usually tactile maps are used to show and explain educational content such as geographic maps, geometric figures, schemes and so on. According to the desired aims, the maps could be designed and developed in different ways. In some cases a Web-based map accessed via screen reader could be enough, if alternative contents are provided. For other goals, a more specific map accessed via new user interaction might be more suitable (e.g., by using a touch screen).

\subsection{Important Map Features}

The main basic abilities required to a user when exploring a map are:

1. Recognition of map borders and pinpointing one's own position within or outside the map. Orientation on the map respects the cardinal points $(\mathrm{N}, \mathrm{S}, \mathrm{E}, \mathrm{W})$.

This requires knowledge of the measurement system.

2. Identifying a point on the map (target). Identifying a destination. Identifying the path between source and destination.

These functions can be carried out automatically using a search function that returns textual information. For instance, Google Maps provides a text box for search and getting directions; retrieved information appears in graphic form on the map as numbered drops $(\mathrm{A}, \mathrm{B}, \mathrm{C})$ and related text info appears on the left side, to be explored visually or auditorily.

3. Exploration of additional related info, such as hotels, restaurants or other points of interest close to the destination.

Touching a map may advance user orientation and improve his/her experience. Smart phones and the latest models of laptops offer integrated touch screens that facilitate physical exploration of the maps. Our idea is to design a multimodal UI to make visual maps accessible to blind users, by both auditory and tactile means. 
However, the system must be designed to have little or no impact on sighted users, i.e. keeping the visual user interface unchanged.

Touch is the equivalent to exploration via mouse, so a mechanism is needed to activate the corresponding audio description (or clue) when a finger-press event is caught by the system. Furthermore, identification of the target should be driven by the system with relative measurements from the map borders. Encoding of gestures and touches are necessary to deliver different amounts of content according to user ability.

\subsection{User Interaction}

In order to achieve the abovementioned goals, a multimodal interaction should be considered when designing a Web-based map. When a developer plans a Web map, they should keep in mind the potential modalities by which a user will explore the map. Many studies have investigated in different contexts how gestures and audio channels can be used to make mobile devices more accessible ([26], [27]). Recently, applications by Apple [26] offer a high accessibility level by using touch and audio channel via the screen reader voice-over; thus, multi-approaches for interaction need to be investigated.

The main aspects to consider regarding user interaction can be summarized as follows:

- Textual content read by an assistive technology (i.e. via screen reader) or at least via voice synthesizer or audio labels.

- Audio icons and aural symbols to provide additional brief information. For example when exploring a map, short sounds could be used to inform the user about a specific category of an item (e.g. whether a touched point is a restaurant, hotel, or a city, etc.).

- Touch. To explore the map item by item or to perceive different areas, using tactile material and special pellicles can offer new multimodal modalities of interaction.

- Vibration feedback. Different kinds and intensities of vibration could be used to inform the user about a specific area, such as borders, mountains, etc.

- Gestures. To better explore and interact with a mobile device (e.g. to explore a map), gestures could represent a valuable way for the users to use.

To apply all those features, recent smart phones, tablets or large touch screens can all effectively achieve the desired results. Web-based maps should be designed and developed taking all these means into account.

\subsection{Designing a Web-based Map}

In this section we consider some aspects of designing a Web-based map for various purposes. In the Introduction we mentioned that a map can be used for three kinds of applications:

1. Orientation and movement: usually this type of information is provided visually. To make this functionality accessible, the user is provided with an alternative description (e.g. in textual format). Additional information or elements could 
improve the overview of the map in order to give an idea of the path or map structure. This means, when providing alternative descriptions additional information that is usually perceived visually should be considered in order to add useful content (e.g. by adding short sounds, labels, general description of the map, etc.).

2. Exploration and location of points of interest: providing a way to explore the map point by point (by adding a sound and /or label for each point), by movements through gestures, and so on can provide new ways to explore a map. In this way the user can obtain more information when "touching" a point. Specific materials such as special pellicles could make certain elements or details more accessible.

3. Educational purposes: especially useful for learning shapes, geographic maps, and geometric figures. Vibration and special pellicles could offer specific features to make certain parts more usable. Certain elements, such as borders, important items, and particular areas could be made accessible thanks to new materials and technologies.

\section{A Web-based Map: a Case Study}

We present a case study to illustrate how a specific map-based function could be made accessible. A map is often used for exploring around one point as occurs when searching for a hotel. A booking hotel service very popular in Italy is http://www.venere.com (Fig. 1).

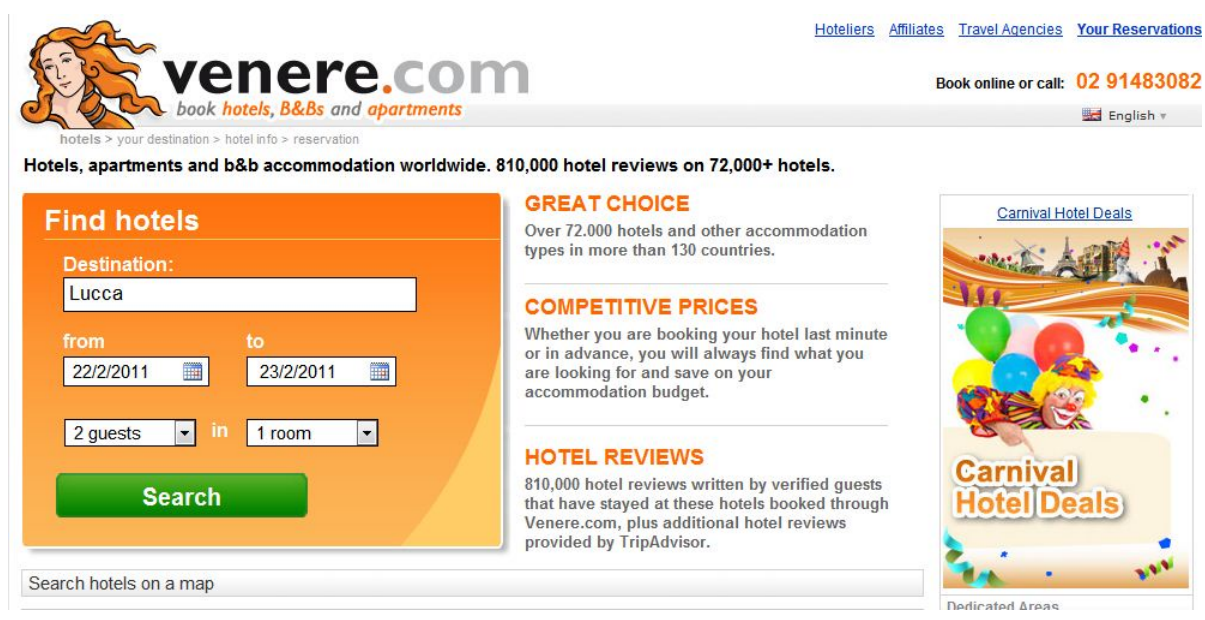

Fig. 1. Venere.com home page

Let us suppose that a person is interested in looking for a hotel in Lucca. After selecting the destination, dates, and room details the query is performed pressing the Search button. Results are available in different ways: on the map, sorted by city areas, budget, stars or accommodation types. 


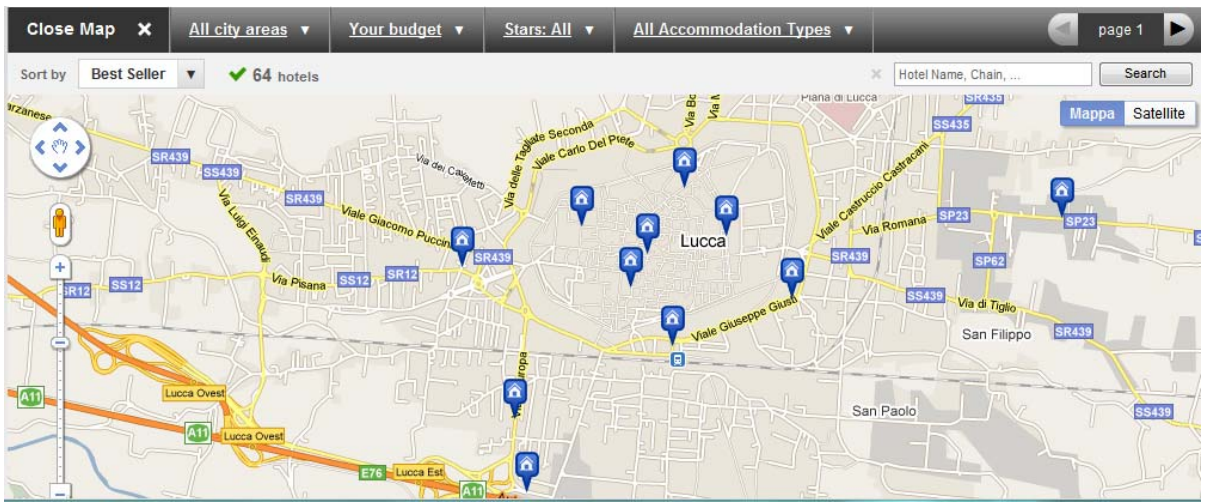

Fig. 2. Query results shown in the map format

On the visual map, hotels are shown as "a house in a blue drop". Two functions are available when using the mouse (Fig. 3):

1. The "on mouse over" function reveals the name of the hotel pointed

2. A left mouse click activates a small frame showing the hotel's details: name (which is a link), stars, price and rating (hotel reviews). Clicking the hotel name the full details (photos, information, location) are shown to encourage the user's booking.

Those two functions can be very useful for quickly locating a hotel that is in the surroundings of a specific point (e.g. a conference venue, a railway station, a airport, a given hotel, etc.). Similar opportunities could be provided through alternative descriptions or textual formats, but they do not provide the same 'semantic' access. For instance, the system could provide a list of the nearby hotels with additional details such as distance, stars and others. That kind of information might not be as usable as exploring a map, since it requires additional effort.

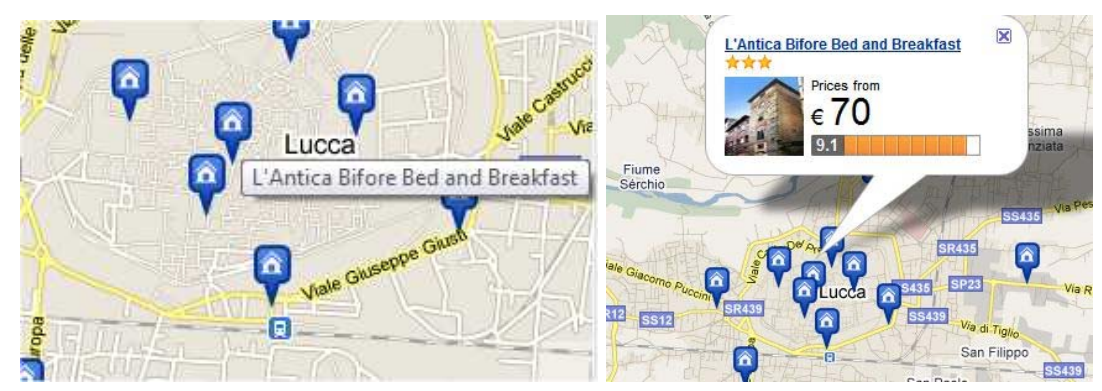

Fig. 3. Results shown in the map format: on the left on mouse over, on the right the mouse click

For making these maps accessible for blind users, those functions should be available even without using a mouse. Touchscreens and other channels (auditory, vibrotactil or Braille) could be valid solutions. 
For instance the "on mouse over" function might be mapped on a single touchscreen click (just one touch) and the mouse single click might be mapped on a double touchscreen clicks (or a double touch). Also, a longer touch might load the page with full hotel details.

In addition, short sounds and audio icons might be a way to rapidly communicate information such as hotel stars or reviews (guests evaluation). Thus, by a single touch the system could communicate the type of the point, i.e. a hotel with a certain number of stars (e.g. through different short sounds) and the name of the item via a label. Other solutions could also be offered by technology (e.g. vibration feedback).

\section{Conclusions}

We discuss possible strategies for making visual maps usable by blind users, exploiting new technologies such as touchscreens and vibration feedback. Maps can be used for various purposes and in different application areas.

In addition, visual maps are increasingly used in websites or for other online services. This field should be investigated by taking into account the potential opportunities offered by new technologies. In this sense, the discussion addressed in this paper could be a starting point for considering issues related to visual maps.

Touchscreens and new ways of providing feedback can create opportunities for interaction modalities for blind users. In the future, there will be increasing use of touchscreens, tablets and smartphones, and this research area should be investigated more thoroughly.

\section{References}

1. Guzzetta, A., Baldini, S., Bancale, A., Baroncelli, L., Ciucci, F., Ghirri, P., Putignano, E., Sale, A., Viegi, A., Berardi, N., Boldrini, A., Cioni, G., Maffei, L.: Massage Accelerates Brain Development and the Maturation of Visual Function. In: The Journal of Neuroscience, 29(18):6042-6051 (2009)

2. W3C Web Content Accessibility Guidelines (WCAG) 2.0, Recommendation W3C (2008) http://www.w3.org/TR/WCAG20/

3. OpenStreetMap. Accessibility http://wiki.openstreetmap.org/wiki/Accessibility

4. Miao, M., Köhlmann, W., Schiewe, M., Weber, G.: Tactile Paper Prototyping with Blind Subjects. Haptic and audio interaction design. In: HAID '09: Proceedings of the 4th International Conference on Haptic and Audio Interaction Design, pages 81-90, Berlin, Heidelberg, (2009)

5. Minatani, K., Watanabe, T., Yamaguchi, T., Watanabe, K., Akiyama, J., Miyagi, M., Oouchi, S.: Tactile Map Automated Creation System to Enhance the Mobility of Blind Persons - Its Design Concept and Evaluation through Experiment. In: K. Miesenberger et al. (Eds.): ICCHP 2010, Part II, LNCS 618, pp. 534-540 (2010)

6. Zeng, L., Weber, G.: Audio-Haptic Browser for a Geographical Information System. In: K. Miesenberger et al. (Eds.): ICCHP 2010, Part II, LNCS 6180, pp. 466-473 (2010)

7. Loomis, J.: Digital Map and Navigation System for the Visually Impaired. Unpublished paper, Department of Psychology, University of California, Santa Barbara (1985) 
8. Spindler, M., Kraus, M., Weber, G.: A Graphical Tactile Screen-Explorer. In: K. Miesenberger et al. (Eds.): ICCHP 2010, Part II, LNCS 6180, pp. 474-481 (2010)

9. Magnusson, C., Brewster, S., Sarjakoski, T., Roselier, S., Sarjakoski, L.T., Tollmar, K.: Exploring Future Challenges for Haptic, Audio and Visual Interfaces for Mobile Maps and Location Based Service. In: LocWeb 2009, April 4, 2009 Boston, MA, USA. (2009)

10.McGookin,D., Cole, R., Brewster, S.: Virtual Navigator: Developing A Simulator for Independent Route Learning. Poster presentation at HAID 2010, Copenhagen, Denmark. (2010)

11.Habel,C., Kerzel, M., Lohmann K.: Verbal Assistance in Tactile-Map Explorations: A Case for Visual Representations and Reasoning. In: Proceedings of AAAI workshop on Visual Representations and Reasoning. AAAIConference 2010, Atlanta, GA, USA (2010)

12. Schmitz,B., Ertl, T.: Making Digital Maps Accessible Using Vibrations. In: K. Miesenberger et al. (Eds.): ICCHP 2010, Part I, LNCS 6179, pp. 100-107 (2010)

13. Sánchez, J.: Mobile Audio Navigation Interfaces for the Blind. Universal Access in HCI, Part II, HCII 2009, LNCS 5615, pp. 402-411 (2009)

14. Miele, J. A., Landau, S., Gilden, D.: Talking TMAP: Automated Generation of AudioTactile Maps Using Smith-Kettlewell's TMAP Software. In: The British Journal of Visual Impairment, vol 24, num. 2, ISSN 0264-6196 (2006)

15. Marsto, J. R., Miele, J., Smith, E.: Large Print Map Automated Production (LPMAP). In: Proceedings of the 23rd International Cartographic Conference, Moscow, Russia (2007)

16. Kulyukin, V., Marston, J., Miele, J., Kutiyanawala, A.: Automated SVG Map Labeling for Customizable Large Print Maps for Low Vision Individuals. RESNA Annual Conference Las Vegas, Nevada (2010)

17. Wagner, A. B.: Collaboratively Generated Content on the Audio-Tactile Map. In: K. Miesenberger et al. (Eds.): ICCHP 2010, Part I, LNCS 6179, pp. 78-80 (2010)

18. Wang, Z., Li, B., Hedgpeth, T., Haven, T.: Instant Tactile-Audio Map: Enabling Access to Digital Maps for People with Visual Impairment. ASSETS'09, Pittsburgh, Pennsylvania, USA (2009)

19.Brock, A., Truillet, P., Oriola, B., Jouffrais, C.: Usage of Multimodal Maps for Blind People: Why and How. ITS'10, Saarbrucken, Germany (2010)

20.Paladugu, D. A., Wang, Z., Li, B.: On Presenting Audio-Tactile Maps to Visually Impaired Users for Getting Directions. CHI 2010, Atlanta, Georgia, USA. ACM 978-1-60558-9305/10/04 (2010)

21.Thomas, K. E., Sripada, S., Noordzij, M. L.: Atlas.txt: exploring linguistic grounding techniques for communicating spatial information to blind users. In: Universal Access in the Information Society, pp. 1-14. DOI 10.1007/s10209-010-0217-5 (2010)

22. McGookin, D., Brewster, S., Jiang, W.: Investigating Touchscreen Accessibility for People with Visual Impairments. NordiCHI 2008: Using Bridges, Lund, Sweden (2008)

23. Crossan, A., Williamson, J., Brewster, S.: Artex: artificial textures from every-day surfaces for touchscreens. CHI 2010, Atlanta, Georgia, USA.ACM 978-1-60558-930-5/10/04 (2010)

24. McGookin, D., Brewster, S.: Eyes-Free Overviews for Mobile Map Applications. MobileHCI'09, Bonn, Germany ACM 978-1-60558-281-8 (2009)

25. Gustafson, S., Baudisch, P., Gutwin, C., Irani, P.: Wedge: Clutter-Free Visualization of OffScreen Locations. CHI 2008, Florence, Italy (2008)

26. Apple. Accessibility. http://www.apple.com/accessibility/

27. Barbara: Ghiani G., Leporini B., Paternò F. Vibro-tactile Feedback to Aid Blind Users of Mobile Guides. In the Journal of Visual Languages and Computing (JVLC), special issue "on Multimodal Interaction Through Haptic Feedback", Volume 20, number 5 (V20N5), October 2009. 